\title{
TELEPSYCHIATRY IN THE TIME OF THE COVID-19 AND EARTHQUAKE IN ZAGREB AS ODYSSEUS BETWEEN SCYLLA AND CHARYBDIS
}

\author{
Daniela Šago ${ }^{1,2}$, Višnja Martić ${ }^{1}$, Dominik Šmida ${ }^{1}$, Nina Mayer $^{1}$, Vanja Lovretić $^{1,2}$ \& Igor Filipčić $^{1,3,4}$ \\ ${ }^{I}$ Psychiatric Hospital "Sveti Ivan", Zagreb, Croatia \\ ${ }^{2}$ Faculty of Medicine, Josip Juraj Strossmayer University of Osijek, Osijek, Croatia \\ ${ }^{3}$ Faculty of Dental Medicine and Health, Josip Juraj Strossmayer University of Osijek, Osijek, Croatia \\ ${ }^{4}$ School of Medicine, University of Zagreb, Zagreb, Croatia
}

received: 14.6 .2020

revised: 7.9 .2020

accepted: 29.10 .2020

\begin{abstract}
SUMMARY
In this paper we would like to reveal some of the many challenges during the time of the twofold simultaneous trauma; the COVID-19 pandemic and the devastating earthquake in Zagreb. We described the functioning mode of two Day Hospitals for Early Intervention and Psychotic Disorders at Psychiatric Hospital "Sveti Ivan" during the outbreak of the COVID-19 pandemic. We tried to find ways to ensure the necessary continuation of treatment and to provide continuity in times of uncertainty. The vulnerable group of people treated for mental illnesses faced exceptional psychological demands and was in need for care in these moments. Telemedicine, more specifically telepsychiatry, through online therapy and telephone communication made it possible for people treated for psychotic disorders not to feel isolated and rejected. Health care professionals, in a state of distress themselves, should understand, support, be able to reduce anxiety, and provide stability and constancy. The psychotherapeutic approach and the capacity for mentalization allowed us to turn challenges into opportunities. Rapid changes without delay extended our scope of practice in these extraordinarily difficult times.
\end{abstract}

Key words: COVID-19 - earthquake - group psychotherapy-mentalization - telemedicine

$* * * * *$

\section{INTRODUCTION}

The outbreak of the COVID-19 pandemic has changed the world overnight confronting us with extraordinary psychological demands. Some measures, such as psychological support, public education, population mobility control, medical isolation, and social distancing, have proven to be effective epidemiologically (Qiu et al. 2020). The implementation of strict quarantine measures has kept a large number of people in isolation and affected many aspects of people's lives (Qiu et al. 2020). In Zagreb where we are stationed, during this pandemic there was an earthquake of magnitude 5.5 on the Richter scale which caused a lot of devastation in the town center, leaving people homeless and in a state of acute stress. The additional distress caused by an earthquake brought some new, but contradictory measures. It was difficult to reconcile the diametrically opposite recommendations at the same time; social distancing, which meant staying home, and going out because of the risk of possible following earthquakes, which meant social gathering. We were given conflicting messages, as is the case with double-entry bookkeeping. For a brief moment in time, we all experienced a psychotic experience filled with fear, uncertainty, and double bind messages. We felt like Odysseus passing between Scylla or COVID-19 and Charybdis or the earthquake. From this straitjacket, we found the path of harm reduction.
Isolation, fear of contagion, stigmatization, frustration, anxiety, insomnia, depression, impaired daily functioning, limited social contact, inadequate health care these were all new challenges to which the general population, including therapists, had to adjust. It is unknown how the new challenges affected patients who due to their illnesses had already faced similar adversities in their lives and have a reduced capacity to adapt. For now, we can only speculate on how social distancing and isolation will affect the mental state (Jakovljević et al. 2020, Kuper-Smith et al. 2020, Lima et al. 2020, Qiu et al. 2020, Torales et al. 2020, Yao et al. 2020).

Findings of the recent study suggest more attention needs to be paid to vulnerable groups, especially for psychological first aid during major disasters, potentially delivered through telemedicine (Duan \& Zhu 2020). These comprehensive aids and targeted interventions should be built to reduce psychological distress and prevent further mental health problems.

The emerging situation required the therapist's flexibility and resourcefulness. We communicated more intensely by email or phone with primary care physicians, informing them of changes in pharmacotherapy, finding replacement medicines in case of a lack of a medicine on the market, adding oral therapy instead of depot therapy in cases of self-isolation, treated comorbidities and occasionally somatic conditions if primary care physician was not available and so on (Filipčić et al. 2018). 
We faced the fear of getting infected either by patients or by colleagues in the health care system who were at the forefront. We also feared that we might be asymptomatic carriers of the virus and that we might infect patients who come to us with full confidence or any of our colleagues which meant that the virus would spread within the healthcare system.

\section{THERAPEUTIC PROGRAM AT THE DAY HOSPITALS}

The drastic changes took place overnight and we, as well as our patients, did not have time to gradually adjust. The COVID-19 epidemic and earthquake hit us during our regular work at the Day Hospital for Early Intervention and the Day Hospital for Psychotic Disorders. Two groups of about 15 patients homogeneous regarding diagnosis (prodrome, first episode psychosis, psychotic disorder) and age (late adolescence, young adults) regularly attend both Day Hospitals (Šago et al. 2018, 2019). The therapeutic program of the Day Hospitals comprises psychodynamically oriented group psychotherapy, multi-family groups, art therapy, cognitive behavioral workshops, metacognitive training, psycho-education, occupational therapy, socio-therapy and recreational therapy, nutrition workshops, and workshops with a social worker in a supportive and endorsing environment (Šago et al. 2018). Activities are carried out by a multidisciplinary team of psychiatrists, psychologist, nurses, nutritionist, social worker and occupational therapist (Šago et al. 2018). All therapists have psychotherapy education. To be effective, therapists should have free mental space to process and metabolize their patients' content. In these times when we are overwhelmed by information, including fake news, unprocessed fears of our own have diminished our mental capacity.

The basic principles of our group work at Day Hospitals, such as supportive social environment, accessibility, stability and constancy were disrupted. We sought to bridge the gap between the two extremes. Intensive daily psychotherapy and psychosocial treatment at Day Hospitals has been replaced overnight by social isolation and the collapse of the normal functioning of the health care system and public transportation. National health authorities paid little attention to the practical implementation of psychological interventions in these situations, so we chose a variety of telepsychiatry modalities to provide assistance, treatment and availability to those who need support (Duan \& Zhu 2020, Fagiolini et al. 2020, Flodgren et al. 2015, Zhou et al. 2020). Considering various aspects, including patients' and therapists' capabilities, familiarity with new online technologies, socioeconomic status, previous experience of therapists, we opted for Skype therapy, and for those patients who were unable to do so, we used regular telephone support and consultation (Fagiolini et al. 2020, Flodgren et al. 2015, Liu et al. 2020, Wright \& Caudill 2020). The challenges have given us the opportunity to grow and develop personally, both therapists and patients (Shalev \& Shapiro 2020).

\section{TELEPSYCHIATRY}

It has been a debate for years if telepsychiatry is as good as in-person visits even though it has greater flexibility (Ćosić et al. 2020). Telepsychiatry requires adaptation from both the therapist and the user (Matačić 2019). Particularly in these times of uncertainty, it was difficult for therapists to provide a secure and stable setting, when we were aware that mobilization of healthcare professionals meant that we may already be working in another workplace tomorrow if the need arises. On the other hand, we do need to adapt to modern times with more and more people spending more time online and developing new pathology such as Hikikomori (Vukojević \& Silić 2018).

As with any new endeavor, it was not without difficulty. In the psychodynamically oriented online group, we made a few changes compared to on-site groups. Patients were divided into smaller groups of 6 (including the therapist) to ensure better communication and to overcome technical difficulties of online platforms.

Adjusting online group psychotherapy treatment with patients going through or recovering from their first episode of psychosis started with explaining the same rules and conditions necessary for such a group to function. The task of the therapist is to provide an adequate setting such as the known length of the group, who initiates online meeting, the exact start time of the group, and the rules for speaking up (Matačić 2019). The therapist instructs all members of the group to find a safe, soundproof space in the house (sometimes even bathroom), to use headphones for securing the privacy of group members from other members of the household, to fix the screen in case of using a smartphone, to take care of the background noise, adequate lighting etc.. This also includes the use of functioning electronic devices that can support internet connection and communication platforms. Since most of these patients are of a younger demographic, the only challenge which appeared was the social and interpersonal aspect in which they had to empathize with other patients to understand how their behavior might affect other people from the other side of the screen. This manifested in few ways: some patients walked with their phones around the house, some were lying down on their beds, and some were smoking, eating, drinking or even had construction workers due to earthquake at the same time in their home or children walking by. It was necessary to alert their attention to these things but even then, they kept repeating some of the behaviors from time to time. 
Some of them were allowed to do so since it seemed it made a bigger intrusion to prohibit smoking or lying down then it was to let it occur. This challenge corresponded to creating a safe space as well because some patients seemed to have a greater problem exposing their vulnerabilities or even speaking up without directions from the therapist. It seemed as though they were more focused on the therapist than on other members of the group. In the beginning, both patients and the therapist needed time to adjust to the new setting or "the lay of the land" - when to speak, how not to talk over other members, what to disclose, how to hear one another and be heard. The beginning was a period of increased anxiety, for both the patients and the therapist. Physical distance reduced personal contact and not being able to observe nonverbal communication as well as the idea that real containment of difficult emotions and topics was not possible, heightened anxiety in the therapist, which had to be metabolized not to affect patients. When working with groups of psychotic patients the technique is modified, and in online groups there was a need for further modification (Ivezić et al. 2017). The therapist assumed a more active role. Therapist's approach in the beginning was from a more supportive stance - how they feel regarding isolation or the earthquake, what their thoughts are, how they organize their day and so on. In the beginning, therapists were reluctant to open up topics of suicidality, or psychoticism, and it was the patients who showed that they were ready to talk about all topics. In time, members of the group became more comfortable in online groups, their initial anxiety in the new setting was diminished and they became more active, opening up about their fears and emotions, giving other members support and advice, from everyday activities to dealing with difficult emotions. With our patients what occurred frequently through the following weeks was that they emphasized the earthquake as traumatic or fear inducing but not the pandemic as much. Moreover, they verbalized that the pandemic either suited their lifestyle or that it was familiar to them. Especially, the social isolation and deprivation aspect of it showed the world how they feel most of the time. Some of the patients saw this as a comforting thing but as time passed some noticed an increase of some negative symptoms and symptoms of depression and anxiety, mostly as a result of social isolation and an unpredictable future which directly impacts their sense of meaning which is often focused on an occupation or a paying job. Better functioning members were able to find a new perspective in this "new normal" which led to personal growth, and they were able to share that new perspective with other group members (Marčinko et al. 2020). Patients often mentioned how important the continuation of contact was for them, it gave them a sense of belonging, of not being alone, of hope that "the world will go on" and that even the worst situations can be overcome (Mayer et al. 2017).
A surprising new sensation that occurred was desensitization of the therapist that occurred at the beginning of the online therapy program with patients and consequential working overload which resulted in steady signs of burnout. Through time it was noticed that being in contact online individually or in groups with patients becomes tiring more quickly than working face to face. What occurs are psychosomatic symptoms like headaches, tired eyes or fatigue and a lack of need for other contacts. Psychosomatic French school attributed it to a deficiency in mentalization, linking concept of operative thinking (pensée opératoire) with vulnerability to somatic illness (Marty \& de M’Uzan 1963, Marty 1991, Šago \& Babić 2019).

Those with whom we were only in contact via telephone regretted the lack of a Day Hospital program, groups, inquired about others, created parallel communication channels and groups. This would be considered resistance by analytic terms, but in the context of people with psychotic experiences who feel isolated and lonely, we also supported this form of community formation.

\section{CONCLUSION}

Even though we did not know what to expect regarding patient compliance, we hoped for the best. Eventually, compliance measured by attendance at sessions ended up above expectations. In part, this is due to already developed group cohesion and partly due to good preparation. Challenges that appeared were diverse and concerned many aspects. Some of them were: the context of an online psychotherapy group, using the necessary technology, containment of emotional reactions without speaking over patients, setting boundaries with limited nonverbal communication, providing a safe space online, desensitization and burnout because of artificial contact, complicated and novel aspects of simultaneous traumatic events, social isolation and consequential increasing of symptoms. There are probably many more that are still unrecognized and will present themselves some time later which could be subject to future research.

\section{Acknowledgements: None.}

\section{Conflict of interest: None to declare.}

\section{Contribution of individual authors:}

Daniela Šago, Višnja Martić \& Dominik Šmida were involved with paper design, literature searches, writing and multiple edits of the manuscript drafts.

Nina Mayer, Vanja Lovretić \& Igor Filipčić were involved with paper design, manuscript preparation, and reviewed the manuscript drafts.

All authors made substantial contributions to conception and design; reviewed the manuscript and have approved the submitted version. 


\section{References}

1. Ćosić K, Popović S, Šarlija $M$ \& Kesedžic I: Impact of human disasters and COVID-19 pandemic on mental health: potential of digital psychiatry. Psychiatr Danub 2020; 32:25-31. https://doi.org/10.24869/psyd.2020.25

2. Duan L\& Zhu G: Psychological interventions for people affected by the COVID-19 epidemic. Lancet Psychiatry 2020; 7:300-302. https://doi.org/10.1016/S2215-0366(20)30073-0

3. Fagiolini A, Cuomo A \& Frank E: COVID-19 Diary From a Psychiatry Department in Italy. J Clin Psychiatry 2020; 81. https://doi.org/10.4088/JCP.20com 13357

4. Filipčić I, Šimunović Filipčić I, Grošić V, Bakija I, Šago $D$, Benjak $T$ et al.: Patterns of chronic physical multimorbidity in psychiatric and general population. J Psychosom Res 2018; 114: 72-80. https://doi.org/10.1016/j.jpsychores.2018.09.011

5. Flodgren $G$, Rachas A, Farmer AJ, Inzitari $M \&$ Shepperd S: Interactive telemedicine: effects on professional practice and health care outcomes. Cochrane Database Syst Rev 2015; 9:CD002098. https://doi.org/10.1002/14651858.CD002098.pub2

6. Ivezić SŠ, Stijačić D, Jendričko T, Biočina SM, Petrović BR, Grah $M$ et al.: Guidelines for individual and group psychodynamic psychotherapy for the treatment of persons diagnosed with psychosis and/or schizophrenia. Psychiatr Danub 2017; 29:432-440

7. Jakovljević $M$, Bjedov $S$, Jakšić $N$ \& Jakovljević I: COVID-19 pandemia and public and global mental health from the perspective of global health security. Psychiatr Danub 2020; 32:6-14.

https://doi.org/10.24869/psyd.2020.6

8. Kuper-Smith BJ, Doppelhofer L, Oganian Y, Rosenblau G \& Korn CW: Optimistic beliefs about the personal impact of COVID-19. PsyArXiv 2020. https://doi.org/10.31234/osf.io/epcyb

9. Lima CKT, Carvalho PMM, Lima IAAS, Nunes JVAO, Saraiva JS, de Souza RI et al.: The emotional impact of Coronavirus 2019-nCoV (new Coronavirus disease). Psychiatry Res 2020; 287:112915. https://doi.org/10.1016/j.psychres.2020.112915

10. Liu S, Yang L, Zhang C, Xiang YT, Liu Z, Hu S \& Zhang $B$ : Online mental health services in China during the COVID-19 outbreak. Lancet Psychiatry 2020; 7:17-18. https://doi.org/10.1016/S2215-0366(20)30077-8

11. Marčinko D, Jakovljević M, Jakšić N, Bjedov S \& Mindoljevic Drakulic A: The importance of psychodynamic approach during COVID-19 pandemic. Psychiatr Danub 2020; 32:15-21. https://doi.org/10.24869/psyd.2020.15
12. Marty P \& de M'Uzan M: La "pensee operatoire". Revue Francaise de Psychanalyse 1963; 27:1345-56

13. Marty P: Mentalization et Psychosomatique. Laboratoire Delagrange, Paris, 1991

14. Matačić S: Cybertherapy: on feasibility and infeasibility of remote psychoanalytic therapies. Psihoterapija 2019; 32:195-215. https://doi.org/10.24869/psihei.2018.195

15. Mayer N, Petrović BR, Grah M, Lovretić V \& Filipčić I: Treatment of patients in early phase of psychosis on psychotherapeutic inpatient unit - presentation of the therapeutic programme and evaluation of some aspects. Psychiatr Danub 2017; 29:447-51.

16. Qiu J, Shen B, Zhao M, Wang Z, Xie B \& Xu Y: A nationwide survey of psychological distress among Chinese people in the COVID-19 epidemic: implications and policy recommendations. General Psychiatry 2020; 33:e100213. https://doi.org/10.1136/gpsych-2020-100213

17. Šago D \& Babić G: Roots of Alexithymia. Archives of Psychiatry Research-2019; 55:71-84. https://doi.org/10.20471/may.2019.55.01.06

18. Šago D, Filipčić I, Lovretić V \& Mayer N: Day hospital for early intervention for individuals with psychotic disorders. Psychiatr Danub 2018; 30:192-197

19. Šago D, Lovretić V, Habuš K, Ivezić E, Bogović Dijaković $A$, Đogaš VV \& Filipčić I: Improving the quality of life during treatment in the Day hospital for early intervention in Psychiatric Hospital "Sveti Ivan". Psychiatr Danub 2019; 31:190-195

20. Shalev D\& Shapiro PA: Epidemic psychiatry: The opportunities and challenges of COVID-19. Gen Hosp Psychiatry 2020; 64:68-71. https://doi.org/10.1016/j.genhosppsych.2020.03.009

21. Torales J, O'Higgins M, Castaldelli-Maia JM \& Ventriglio A: The outbreak of COVID-19 coronavirus and its impact on global mental health. Int J Soc Psychiatry 2020; 2076402091521. https://doi.org/10.1177/0020764020915212

22. Vukojević J, Silić A: The future of psychiatry and the story of two worlds. Asian J Psychiatr 2018; 35:3. doi:10.1016/j.ajp.2018.04.027. Epub 2018 Apr 20

23. Wright JH \& Caudill R: Remote Treatment Delivery in Response to the COVID-19 Pandemic. Psychother Psychosom 2020; 1-3. https://doi.org/10.1159/000507376

24. Yao H, Chen JH \& Xu YF: Patients with mental health disorders in the COVID-19 epidemic. Lancet Psychiatry 2020; 7:e21. https://doi.org/10.1016/S2215-0366(20)30090-0

25. Zhou X, Snoswell CL, Harding LE, Bambling M, Edirippulige S, Bai $X$ \& Smith AC: The Role of Telehealth in Reducing the Mental Health Burden from COVID-19. Telemed J E Health 2020; 26:377-379. https://doi.org/10.1089/tmj.2020.0068

Correspondence:

Daniela Šago, $M D$

Psychiatric Hospital "Sveti Ivan"

Jankomir 11, 10090 Zagreb, Croatia

E-mail: daniela.sago@pbsvi.hr 